\title{
Reparameterizing Tectonics Perception on Planar Material-Design
}

\author{
Stephanus Evert Indrawan ${ }^{1}$, Gervasius Herry Purwoko², \\ Tri Noviyanto P. Utomo ${ }^{3}$ \\ Interior Architecture Department, Ciputra University \\ UC Town-Citraland Surabaya ${ }^{1,2,3}$ \\ Email: sindrawan@ciputra.ac.id ${ }^{1}$; gpurwoko@ciputra.ac.id²; tommy@ciputra.ac.id ${ }^{3}$
}

\begin{abstract}
Indonesia is known as a country rich in types of building materials and technologies inherited from generation to generation. Along with the passage of time appears the computer as a human tools. However during the materials processing this computational approach is still separated. Computers are still used as tools for drawing and not used as design tools in the design thinking process. Computational design has an ability to integrating the design focus from the material side, structure, and formation associated with digital fabrication. This paper focuses to divide concepts of tectonics as general and relate them to the understanding of digital perception. This paper also presents the results of a study that has involved digital perception in the study of planar materials and waffle structure systems from the early stages to the model of construction. Plywood is the only material used, made with milling machines and built by students. This process introduces students to different experience of the design process. Computational design makes possibilities to integrating the design focus from the material side, structure, and formation associated with digital fabrication.
\end{abstract}

Keywords: computational design, digital fabrication, digital perception, design tools

\section{INTRODUCTION}

Architects are actively involved to design a better living environment. It still cannot be avoided, modern production demands for effectiveness and efficiency. This situation have divided the architectural process into two major domains: "design" which represents ideas and "constructs" that represent jobs field (Armand, 2014). Architectural process cannot be separated from tectonics skills and perception. Based on Historical references. Tectonics word or Tekton originally from Greek word that means carpenter or builder. Tectonics is a concept that defines the relationship between architectural design and its structures and materials. The variety of the tectonics of Nusantara skill are highly influent the history of Indonesian Architecture. The skill of builders that inherited from generations who skillfully carve, sculpts, and build an Architecture. The first 
element of tectonics is material. Tectonics cannot be separated from material that being used. The Architecture of Nusantara generally was made from wood, stone, and bricks. During the colonial era and industrial revolution era, concrete become the main material for modern and colonial buildings. On the other hand, Indonesia is world-known country that has many kind of material resources and technology. From this explanation can be seen there are tectonics of Nusantara before colonialism era and modern tectonics during and after colonialism era. Modern tectonics itself is divided from before the digital era and the digital era. According to Oxman, the relationship between architecture and structure changes over time influenced by periods. Nowadays, computer are becoming tools for architect during drawing production process but during conceptual stage most of designer still rely on pencil and paper. Fabrication and production process are separate from the process of design. This research proposes alternative to designer to explore larger design possibilities to achieve design result.

\section{Design domain and CAD support}

The complexity of design and design problem requires designer to produce many design alternatives. Most of this activity happens during initial stage, pencil and paper become the most important tools that chosen by most of designers. During this stage, most of commitments have been decided and remarkable improvements cannot be made. There are some logical explanation that prevent involving CAD in initial stage of design.

1. The Development of design.

In the creative process, the design exploration stage depends on the results of previous exploration. Creative design is based on reflection, reaction, criticism, and inspiration taken from the process itself. The exploration of the designer relies heavily on understanding the problem and potential solutions to the problem. Oxman Defines 'conceptual emergence' as a search for 'visual fit' images stored in the designer's mental image memory and the way the designer maps of these images into a formal-configurational schema. Designer imagination is needed to identify design solutions by departing from abstract things.

2. The importance of sketch.

Sketching has an ability to stimulate creative process in concept development process and helping to generate it.

3. The nature of design process is chaos and unstructured.

There are some requirement that need to meet for CAD to support designers during conceptual stages: 1) Minimum requirements and disturbance on designs process; 2) Operational flexibility for designer; 3) Be able to manage chaotic design stages; 4) Stimulating designers creativity; 5) Efficiency to translate design content to detailed designs phase. 


\section{The Evolution of Tectonics}

Tectonics word or Tekton derived from Greek word that means carpenter or builder. Tectonics is a concept that defines the relationship between architectural design and its structures and materials. The relationship between Architectures and structures was changing from time to time influenced by its periods (R.Oxman, 2010). There are concept of tectonics that defines its relation to informed tectonics.

1. Vernacular Tectonics was the origin of tectonics expression.

Vernacular design always expressing the essence of material technology. In the vernacular, traditional material technologies evolve to become buildings system. The system are strongly related to material origin and construction process. The expression of vernacular tradition are an implicit "poetics" or explicative theory. The Traditional House of Japan is a good example of vernacular tectonics, providing an essential expression of construction potentials of material.

2. Tectonic as Culture: prioritizing building and construction, since the $19^{\text {th }}$ century and through modern periods can be seen that the theory of tectonic evolve through the nature of design. According to Frampton "the poetry of constructions" is the foundation of modern architecture and shape, structures and materials still interpretated separately. This condition brings a closer exploration of tectonics to contemporary definition in which fabrication and manufacturing are viewed as parametric systems.

3. Digital Tectonics as virtual and physical materiality.

There are various different approaches of digital tectonics in the last decade: 1) digital tectonics as virtual materiality, a virtual computational space is an accommodation of material representation. 2) Digital tectonics as physical materiality, digital tectonics was related to dynamic factors of motion, information, generation and fabrication. 3) Digital tectonics as fabricated materiality. 4) Digital Tectonics as structured materiality, digital technologies make technological possibilities more affordable 5) Digital tectonics as digital form-finding and morphogenetic processes, related to form-finding processes and in-nature. 6) Digital tectonics as adaptive materiality, adaptive building required an adaptive material by system performance of material. (Oxman, 2012) 


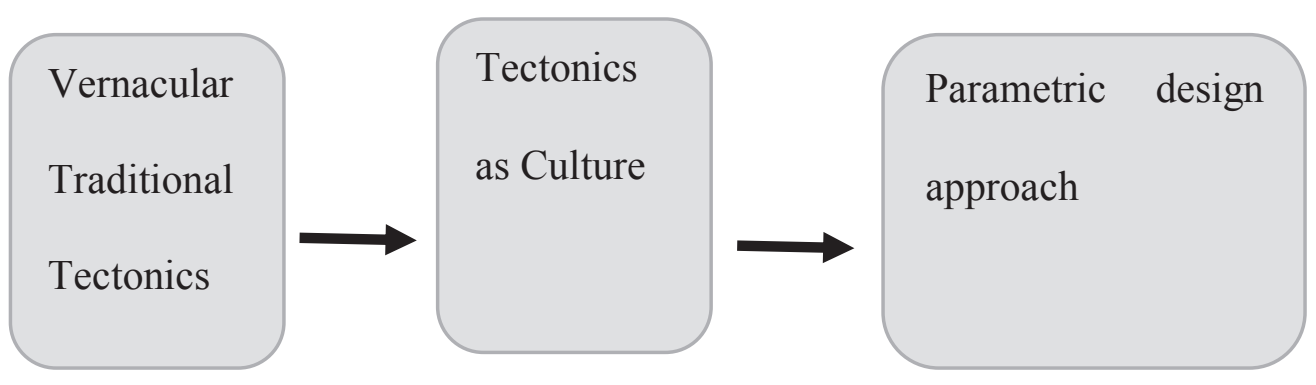

Figure 1. Scheme of Vernacular Tectonic to Informed Tectonics concept

\section{Research Frameworks: Generative Design and Digital Fabrications}

From previous explanation can be identified that the usage of digital tools should not hinder the creative freedom. The work flow should be designers oriented in order to see how the design result fit in design problem. The following explanation we present the theoretical description of generative design along with digital fabrication implementation. Generative design and digital fabrication method are implemented for pavilion that made from planar material. Generative design here is designer driven, parametrically constrained design exploration process. Generative design can be applicable to both parametric and procedural design.

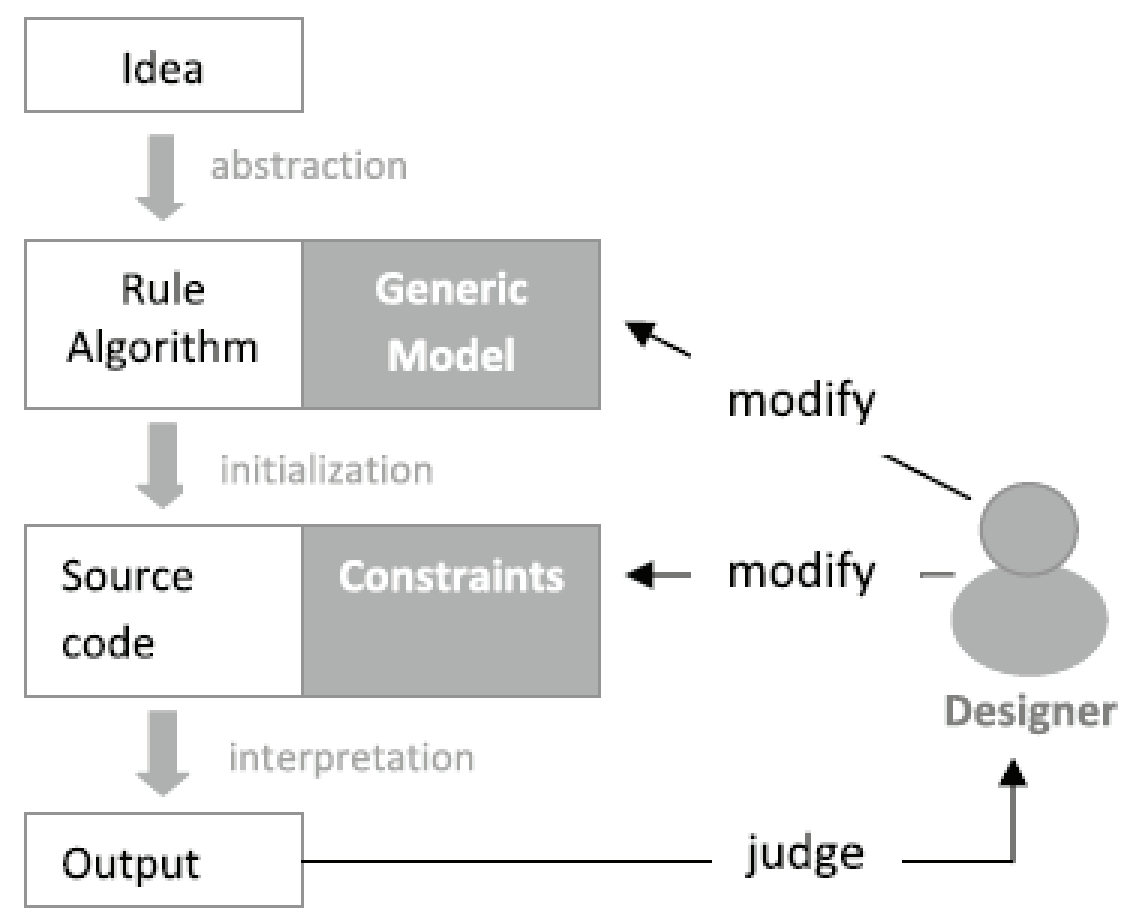

Figure 2. Generative design process 
The aim of Generative design according to Shea: "generative design systems are aimed at creating new design processes that produce spatially novel yet efficient and buildable designs through exploitation of current computing and manufacturing capabilities". The process of generative design consist of this following components:

1. The diagram of design.

2. A medium to create alternatives or design possibilities.

3. A medium to select considered outcomes.

The process of generative design can be described into Configuration variation, Performance Metric, and Decision making response. The Generative Design Method built by seven components:

1. Genotype - is composed of a generic parametric CAD model, list of design parameters and their initial value and initial exploration envelope.

2. Phenotype - generated CAD files (that may include build history, built-in relationships, and built-in equations).

3. Exploration envelope - a list of minimum and maximum values of the driving parameters, specifying the limits of the design space to be explored.

4. Design Table - a data table that stores the driving design parameters, their initial values and limits.

5. Design Generation Software - a spreadsheet function, a macro or a CAD plug-in that operates on the design table. It generates random variations of the driving design parameters within limits set by the exploration envelope.

CAD system - is a parametric CAD engine with a transparent and editable build history, preferably with a 3D geometric kernel with capabilities to manage geometric relationships, engineering equations and connect to external design tables.

Performance filters - A pass/fail software filter, that is able to evaluate the performance of generated designs based on preset performance criteria.

The performance may be evaluated directly from the design table or by the CAD's inbuilt evaluation tools or by the use of external analytical software associated with the CAD package. Beside Generative design method, digital production also narrows the gap between representation ideas and forming. Digital fabrication has a capability to document the architecture process through digital design and innovative constructive projects. There are five categories of digital fabrication method that mentioned by Iwamoto, there are:

1. Sectioning, it is a part of constructions history. This method was a usually being used to construct the surface of airplane and shipbuilding industry. The 
shape of airplane and shipbuilding were firstly defined sectionally as a series of construction elements.

2. Tessellation is a collected form that applied on a form or surface. Tessellation are known during ancient Rome and those Byzantine Empire to the screen wall of Islamic Architecture to filter the lights, define space or convey symbolic meanings. Tessellation are strongly related to handcraft culture and digital fabrication gives a different point of view for producing the work of tiling.

3. Folding is a powerful techniques to turn flat surfaces into three dimensional form. Folding structure has an ability for self-support and wide span structure. Folding principles has been embraced by architects for more than fifteen years.

4. Contouring is method to stack flat surfaces to construct $3 \mathrm{~d}$ objects. Digital fabrication enables this method to transcend the idea of handcrafting in unorthodox practices.

5. Forming is a mass production method that applicated into Architectural projects.

\section{Design Implementation}

Cartesian coordinate system can be evolved into UV mapping. UVW coordinates has a different concept compares to XYZ. The UV mapping process involves applying pixels in the image to surface mappings on the polygon. This process normally done "programmatically" by pasting material onto triangular surface (Mullen, 2009). UV only maps material onto texture space rather than geometrical space of objects. Parametric Design approach using the principles of UV Mapping to apply geometry onto surface (Murdock, 2008).

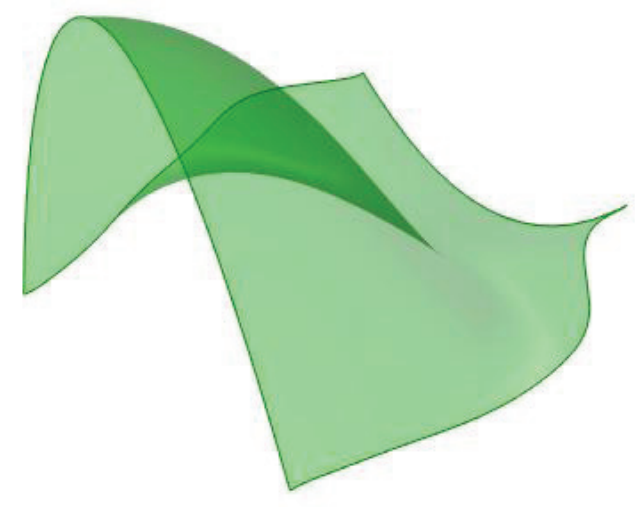

Figure 3. Surface

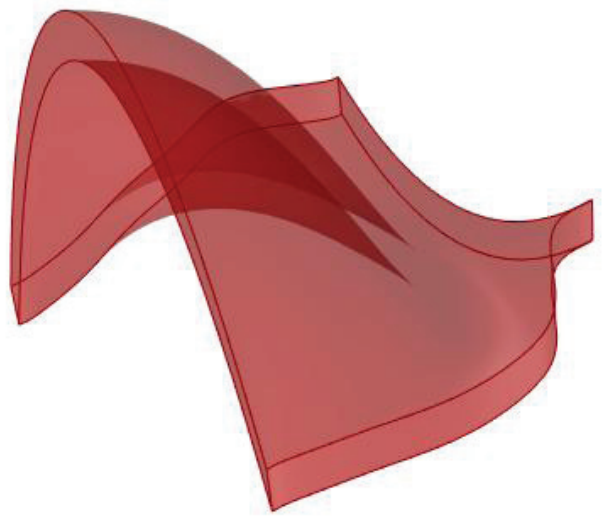

Figure 4. Volume

Designer can produce a digital sketch that represented by surface through parametrical design approach. It is a concept of modeling definition that can be controlled and modified by user. After the surface being modeled, it can be 
extruded to achieve certain volume. The model volume was created through association, definition and variety solving method.

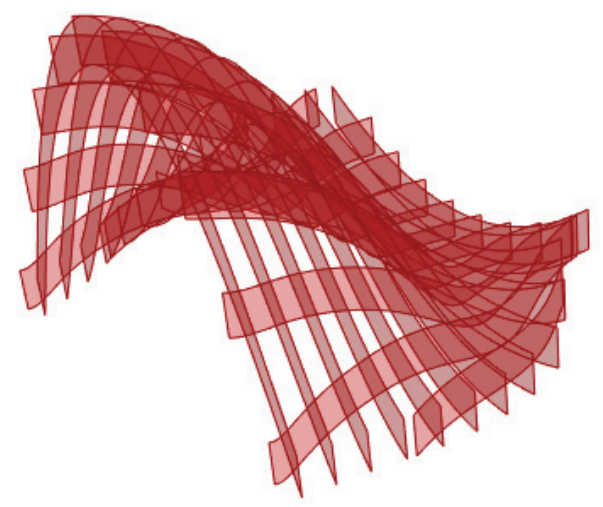

Figure 5. Section

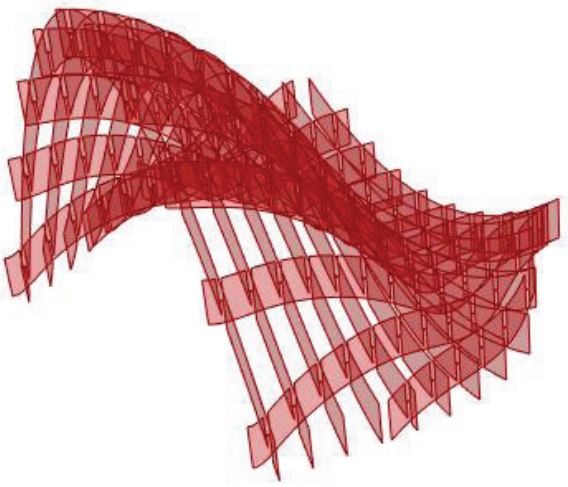

Figure 6. Joinery

The behavior of design is associative, it can be managed by user and modified associatively related to objects. The input-output mapping and mathematical expression are described as mathematical expression which describes the generative process.

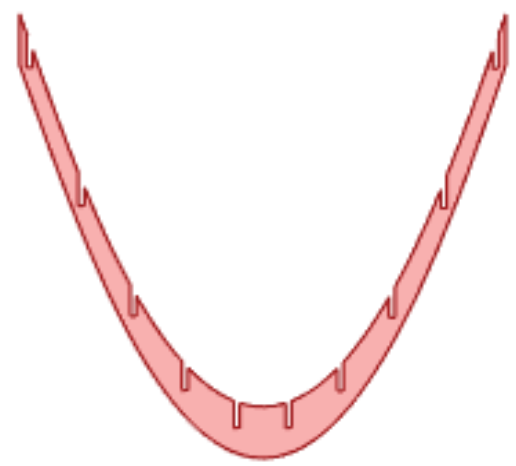

Figure 7. Section

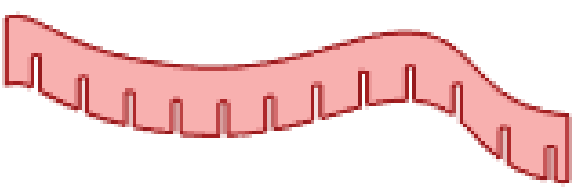

Figure 8. Section

Definition is a concept that shows that objects that have definitions can be processed by a computer by determining input parameters to outputs, either numerically, in objects or visually. Definitions are often considered as methods for updating, but are sometimes hidden from the user through input interpretation automatically. Definitions can be defined by the user through packing object logic on a new object ("feature building" in GC, "clustering" in Grasshopper), with scripting or programming. 


\section{||JCAAS:}

Volume 6 Number 1, June 2019 | p-ISSN 2339-191X, e-ISSN 2406-9760

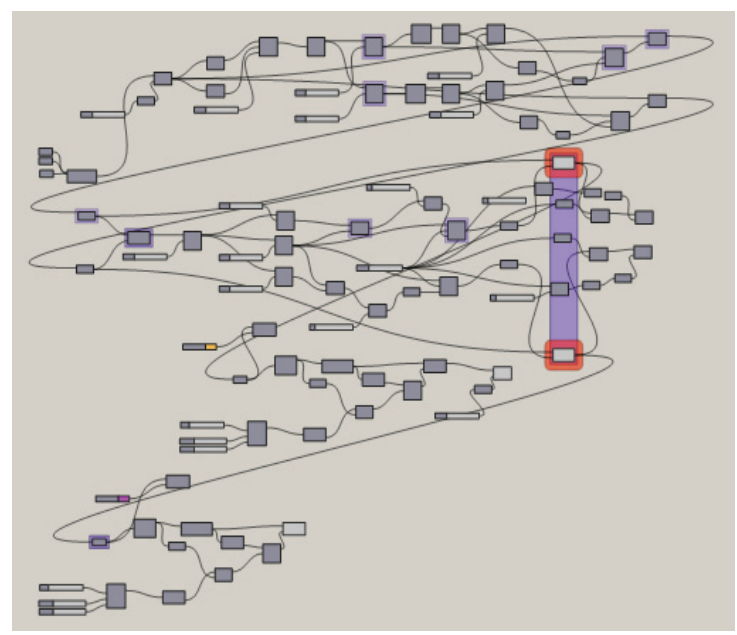

Figure 9. Definition from

The sectioning method that explained by Iwamoto was chosen to make pavilion. This research selects planar material to be the main material for pavilion, because planar material is the common form that found in Indonesian market. The whole process through $\mathrm{CNC}$ machine. Students were involved from initial stage to assembling process.

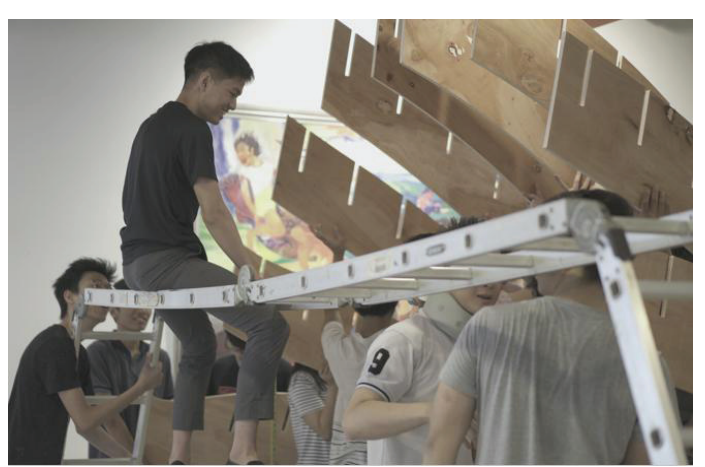

Figure 10. Assembling process

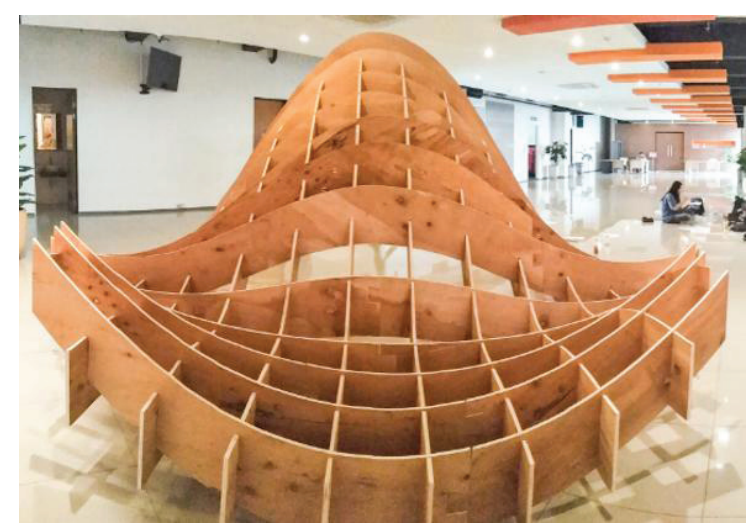

Figure 12. Rear Elevation

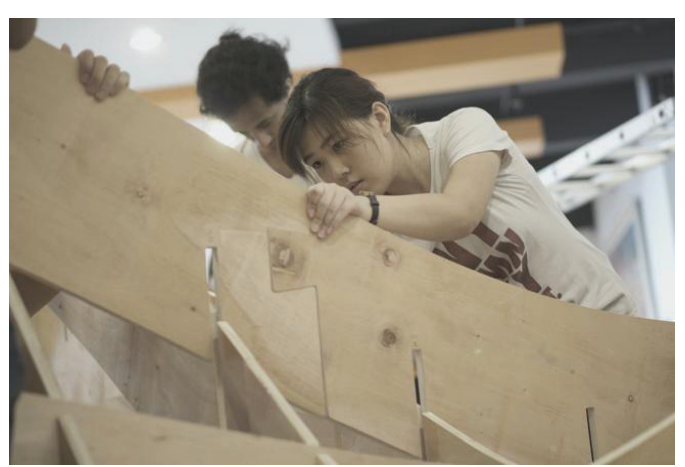

Figure 11. Joinery checking

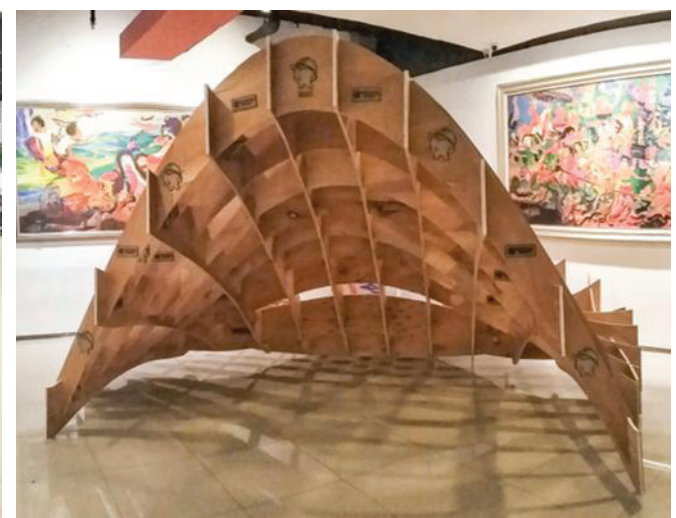

Figure 13. Front elevation 


\section{RESULT AND CONCLUSION}

From explanation and design implementation can be identified that digital tools should followed by digital methods to achieve sketching quality. Digital tools should provide possibilities for designer to work creativily. Generative design manage the unstructured flow of conceptual design process. The evolution of tectonics explain interrelation between tectonics skill, material knowledge to architecture or design objects. This relation was change from time to time and highly influenced by efficiency, effectiveness and preservation purposes. Digital fabrications generally explain the structure relation between conceptual stage to fabrication process and it narrow the gap between design to realization stages.

\section{REFERENCES}

Iwamoto, Lisa. 2010. Architecture briefs. Digital Fabrications: architectural and Material Techniques. http://www.papress.com/html/ book.details.page.tpl?cart $=125777262860796 \&$ isbn $=9781568987903$.

Krish, Sivam. 2011. "A Practical Generative Design Method". CAD Computer Aided Design 43(1): 88-100. http://dx.doi.org/10.1016/j.cad.2010.09.009.

Mullen, T. 2009. MasteringBlender. 1st ed. Indiana: Wiley Publishing.

Murdock, K L. 2008. 3DS Max 2009 Bible. 1st ed. Indiana: Wiley Publishing. Oxman, Rivka. 2012. "Informed Tectonics in Material-Based Design". Design Studies 33(5): 427-55. 